\title{
Prevalence of Diabetes and Dyslipidemia in Hypertensive Young Adults in a Tertiary Care Centre in Nepal, a Cross Sectional Study
}

\author{
Kamal Sharma Lamsal', Keshab R Neupane1, Niranjan Acharya', Dinesh K Lamsal' ${ }^{1}$, Niraj \\ Karmacharya', Deepak Paudel', Rajan Paudel'1, Rabi P Sharma ${ }^{1}$ \\ 'Department of Medicine, ${ }^{2}$ Department of Family Medicine, Civil Service Hospital, Minbhawan, Kathmandu, Nepal
}

\section{Corresponding author:}

\section{Kamal Sharma Lamsal, MBBS, MD}

Department of Medicine, Civil Service Hospital, Minbhawan, Kathmandu, Nepal

Email:kamalslamsal@gmail.com

Submitted : May 7, 2020

Accepted : July 3, 2020

\begin{abstract}
Introduction

Diabetes mellitus (DM) and lipid disorders (LD) in hypertensive patients are associated with increased risk of cardiovascular complications requiring close follow-up and more aggressive treatment. Hence, the present study was done to study the prevalence of diabetes and dyslipidemia in young hypertensive patients i.e. $<40$ years in a tertiary care hospital, Nepal.
\end{abstract}

\section{Methods}

This is a hospital based cross-sectional study carried out in Civil Service Hospital, Kathmandu. Study included 165 patients of $<40$ years who had hypertension, either newly diagnosed or undergoing antihypertensive treatment from September 2018 to September 2019. Blood pressure was measured two times on right upper arm in sitting position at interval of 30 minutes and the average was taken. Fasting Lipid Profile and Fasting Blood Glucose was measured after 12 hours of fasting and the prevalence of dyslipidemia and diabetes were analyzed from the data collected. All calculations and statistical analyses are processed by the SPSS 25.0.

\section{Results}

Among 165 patients included, majority of patients, 107 (64.8\%) were among 31-39 years, followed by 53 (32.1\%) patients among 21-30 years. In total, 98 (59.4\%) were male and 67 (40.6\%) female. The prevalence of diabetes was $24(14.5 \%)$ and pre-diabetes was 49 (29.7\%). The prevalence of dyslipidemia was 125 (75.6\%), high triglyceride being the most common disorder followed by low HDL in 87 (52.1\%). Elevated total cholesterol was found in 53 (32.1\%) patients and 48 (29\%) had high LDL cholesterol level.

\section{Conclusion}

A high prevalence of diabetes, pre-diabetes and dyslipidemia was observed in the hypertensive patients $<40$ years of age.

Keywords: Diabetes, dyslipidemia, hypertension, prediabetes 


\section{INTRODUCTION}

C ardiovascular disease (CVD) is the leading cause of mortality and morbidity in the world and is increasing day by day. The high prevalence of CVD is attributed to multiple modifiable cardiovascular risk factors, such as high blood pressure (hypertension), diabetes, dyslipidemia and obesity. Many of these risk factors tend to cluster and coexist in the same individual. Hypertension and hypercholesterolemia are the most common of these risk factors. ${ }^{1,2}$ The risks associated with concomitant hypertension and dyslipidemia are greater than the sum of the risks associated with hypertension or dyslipidemia alone and such patients are associated with an increased risk of cardiovascular complications requiring followup and more aggressive therapeutic strategies. ${ }^{3}$ The cluster of these risk factors is metabolic syndrome. In 2000, approximately $32 \%$ of U.S. adults had the metabolic syndrome and in the recent years that figure has climbed to $37 \% .^{3}$ In a recent large prospective cohort study, the development of type 2 diabetes was almost 2.5 times as likely in persons with hypertension than in their normotensive counterparts. ${ }^{4}$ The recent studies in Korea also suggest that dyslipidemia; diabetes and hypertension may be developing at even younger ages. ${ }^{5}$

In one study in Nepal, Kashyap Dahal et al studied 38 patients presenting with acute myocardial infarction, among them $44.75 \%$ were hypertensive and $47 \%$ had diabetes. ${ }^{6}$ In another study of ST elevation myocardial infarction (STEMI) in young population in Nepal, Chandra Mani Adhikari et al studied 132 young (aged less than 45 years) STEMI patients, which comprised $10.9 \%$ of total STEMI cases. Among them dyslipidemia, hypertension and diabetes were present in $45.5 \%, 29.5 \%$ and $25.8 \%$ respectively. The burden of such acute cardiac events can be decreased by optimal control of risk factors. Hence, this study was performed to find out the prevalence of diabetes and dyslipidemia in young hypertensive patients in a tertiary care hospital in Nepal. The data can be useful to plan appropriate preventive and therapeutic strategies in those high risk individuals.

\section{METHODS}

This is a hospital based, cross-sectional observational study carried out in Civil Service Hospital, Kathmandu Nepal from September 2018 to September 2019. Approval was taken from Institutional Review Committee of the hospital, IRC protocol No is $05 / 2020$. Written consent was taken from the enrolled participants. Using $n=z 2 p q / d 2$ with $10 \%$ error, sample size is calculated to be 100 . Consecutive sampling method was used and one hundred and sixty five patients were enrolled in the study. Patients included were $<40$ years of age having hypertension, either primary or secondary. They were either newly diagnosed or undergoing treatment for hypertension. All patients $\geq 40$ years of age were excluded from the study. Critically ill patients admitted in Intensive Care Unit were excluded.

Blood pressure was measured using standard technique with mercury sphygmomanometer with appropriate cuff size. The blood pressure was measured two times on right upper arm in sitting position in the interval of 30 minutes and the average of the two was taken. Hypertension was defined as systolic blood pressure $\geq 140 \mathrm{mmHg}$ and/or diastolic blood pressure $\geq 90 \mathrm{mmHg}$. The diagnosis and classification of hypertension was done according to the Seventh Joint National Committee on the Prevention, Detection, Evaluation and Treatment of High Blood Pressure (JNC-7) report. ${ }^{8}$

Fasting Lipid Profile and Fasting Blood Glucose was measured after 12 hours of fasting. Diagnosis and classification of dyslipidemia was done according to National Institute of Health (NIH) guidelines, 2012. ${ }^{9}$ Diabetes was diagnosed if Fasting Blood Sugar (FBS) $\geq 126 \mathrm{mg} / \mathrm{dl}$ \& Impaired Fasting glucose as $\geq 100$ to $125 \mathrm{mg} / \mathrm{dl}$; as per American Diabetes Association (ADA) 2018 guidelines. ${ }^{10}$ Data on a few demographic characteristics of patients, Lipid Profile including Triglycerides, Total cholesterol, Low Density Lipoprotein (LDL) and High Density Lipoprotein (HDL) and Fasting Blood Glucose were collected and the prevalence of Dyslipidemia and Diabetes were analyzed from the data collected. Descriptive statistics was used; the data were analyzed using SPSS 25 software.

\section{RESULTS}

Among the 165 hypertensive patients aged $<40$ years, most of the patients 107 (64.8\%) were between the age group 31-39 years and 53 (32\%) were in the age group 21-30 years and only five patients were $\leq 20$ years of age. In total, 98 (59.39\%) were male and 67 (40.6\%) female.

In the study, $11(6.67 \%)$ were known case of diabetes mellitus and most of them belonged to age group 31-39 (Table 1). However, among the 154 patients with no prior diagnosis of diabetes mellitus, $49(31.81 \%)$ patients had Fasting Blood Glucose $100-125 \mathrm{mg} / \mathrm{dl}$ and $13(8.44 \%)$ patients had Fasting Blood Glucose $\geq 126 \mathrm{mg} / \mathrm{dl}$, in the diabetes range. The overall prevalence of diabetes was $14.5 \%$ (24 out of 165) and pre-diabetes was 29.69\% (49 out of 165).

Out of the 165 study population, 125 (75.6\%) patients had elevated TG level i.e. more than $150 \mathrm{mg} / \mathrm{dl}$ with $63(38.2 \%)$ patients had borderline high, 61 (37\%) patients had high TG level and only 
Table 1. Age versus Diabetes Cross tabulation $(N=165)$

\begin{tabular}{cccc}
\hline \multirow{2}{*}{ Age } & \multicolumn{2}{c}{ Diabetes } & \multirow{2}{*}{ Total } \\
\cline { 2 - 3 } & Yes (\%) & No (\%) & \\
\hline$\leq 20$ & 0 & $5(100)$ & 5 \\
$21-30$ & $1(1.9)$ & $52(98.1)$ & 53 \\
$31-39$ & $10(9.3)$ & $97(90.7)$ & 107 \\
\hline Total (\%) & $11(6.6)$ & $154(93.4)$ & $165(100)$ \\
\hline
\end{tabular}

one patient had very high TG level (table 2). In our study, 53 (32.1\%) patients had elevated total cholesterol level i.e. more than $200 \mathrm{mg} / \mathrm{dl}$ with 36 $(21.8 \%)$ patients having borderline high i.e. 200$239 \mathrm{mg} / \mathrm{dl}$, and 17 (10.3\%) patients having high total cholesterol level i.e. 240 mg/dl or higher (table 3).

One hundred thirty two (80\%) patients had LDL cholesterol level more than $100 \mathrm{mg} / \mathrm{dl}$ with 84 (50.9\%) patients had borderline high (100-130mg/dl) LDL cholesterol and 48 (29\%) had high cholesterol i.e. more than $130 \mathrm{mg} / \mathrm{dl}$. Among high cholesterol levels, 24 (14.5\%) patients had borderline high, $14(8.5 \%)$ patients had high LDL cholesterol level and $10(6.1 \%)$ patients had very high LDL level (Table 4). Regarding HDL cholesterol, 87 (52.72\%) patients had low level with $78(47.27 \%)$ patients in acceptable level (Table 5). The number of patients with low HDL cholesterol levels has been shown to be increasing as the age group increases.

\section{DISCUSSION}

This study was designed to have important data on the prevalence of the two important cardiovascular risk factors: diabetes and dyslipidemia in young hypertensive patients, attending a tertiary hospital in the capital city of Nepal. Out of the study population, 107 (64.8\%) patients belonged to the 31-40-age group followed by 53 (32\%) patients in 21-30 age group and only 5 patients belonged to $\leq 20$ age group, reflecting increasing prevalence of hypertension with age.

Table 2. Age versus Lipid Triglycerides (TG) Cross tabulation $(N=165)$

\begin{tabular}{cccccc}
\hline \multirow{2}{*}{ Age } & \multicolumn{4}{c}{ TG (mg/dl) } & \multirow{2}{*}{ Total } \\
\cline { 2 - 5 } & $<\mathbf{1 5 0}$ & $\mathbf{1 5 0 - 1 9 9}$ & $\mathbf{2 0 0 - 4 9 9}$ & $\mathbf{2 5 0 0}$ & \\
\hline$\leq 20$ & 04 & 1 & 0 & 0 & 5 \\
$21-30$ & 21 & 22 & 10 & 0 & 53 \\
$31-39$ & 15 & 40 & 51 & 1 & 107 \\
\hline Total & 40 & 63 & 61 & 1 & 165 \\
$(\%)$ & $(24.2)$ & $(38.2)$ & $(37)$ & $(0.6)$ & $(100)$ \\
\hline
\end{tabular}

Table 3. Age versus Total cholesterol (TC) Cross tabulation $(N=165)$

\begin{tabular}{ccccc}
\hline \multirow{2}{*}{ Age } & \multicolumn{3}{c}{ TC (mg/d) } & \multirow{2}{*}{ Total } \\
\cline { 2 - 4 } & $<200$ & $\mathbf{2 0 0 - 2 3 9}$ & $\geq 240$ & \\
\hline$\leq 20$ & 5 & 0 & 0 & 5 \\
$21-30$ & 41 & 9 & 3 & 53 \\
$31-39$ & 66 & 27 & 14 & 107 \\
\hline Total (\%) & $112(67.8)$ & $36(21.8)$ & $17(10.3)$ & $165(100)$ \\
\hline
\end{tabular}

In this study, the prevalence of diabetes was 24 $(14.5 \%)$ and pre-diabetes was 49 (29.69\%). Our results are similar to those of Nkatha Meme in Kenya, in which during an 8-year follow-up period, about $14.7 \%$ of the subjects included developed diabetes, while $18 \%$ were pre-diabetic ${ }^{11}$ and of Luders et al. in Germany who found that out of 260 hypertensive patients, 39\% had impaired glucose tolerance and $12 \%$ had diabetes mellitus. ${ }^{12}$ Conversely, A. BachirCherif et al. in Algeria showed higher prevalence of diabetes, comprising $21.8 \%$ diabetes and only $10.9 \%$ impaired glucose tolerance..$^{13}$ This study shows the prevalence of diabetes increases with increasing age which is probably due to the combined effect of body mass index (BMI) and age, as suggested by previous studies. ${ }^{14}$

The prevalence of dyslipidemia was 125 (75.6\%) in our study. Our results are similar to the study done by L.P. Thiombiano et.al., which included 705 patients in Senegal, $61.3 \%$ of cases with hypercholesterolemia. ${ }^{15}$ In Nigeria, Akintunde et al. found out that $58.9 \%$ newly diagnosed hypertensive patients had at least one impaired lipid parameter. ${ }^{16}$ Conversely, lower prevalence was found in study done by A. BachirCherif et al.,which included 3268 hypertensive patients in Algeria. Among them $16.1 \%$ had dyslipidemia. ${ }^{13}$ Zhang et al. in China $^{17}$ and Pedrinelli et al. in Italy ${ }^{18}$ found the prevalence of dyslipidemia to be $16.9 \%$ and $19.1 \%$ respectively in hypertensive patients. In our study, the most common disorder was found to be high TG in 125 patients (75.6\%) followed by Low HDL

Table 4. Age versus LDL cholesterol Cross tabulation $(N=165)$

\begin{tabular}{ccccccc}
\hline \multirow{2}{*}{ Age } & \multicolumn{5}{c}{ LDL (mg/dl) } & Total \\
\cline { 2 - 7 } & $<100$ & $100-129$ & $130-159$ & $160-189$ & $\geq 190$ \\
\hline$\leq 20$ & 2 & 2 & 1 & 0 & 0 & 5 \\
$21-30$ & 15 & 30 & 3 & 3 & 2 & 53 \\
$31-39$ & 16 & 52 & 20 & 11 & 8 & 107 \\
\hline Total & 33 & 84 & 24 & 14 & 10 & 165 \\
$(\%)$ & $(20)$ & $(50.9)$ & $(14.5)$ & $(8.5)$ & $(6.1)$ & $(100)$ \\
\hline
\end{tabular}


Table 5. Age versus HDL cholesterol Cross tabulation $(N=165)$

\begin{tabular}{|c|c|c|c|}
\hline \multirow{2}{*}{ Age } & \multicolumn{2}{|c|}{ HDL } & \multirow{2}{*}{ Total } \\
\hline & $40-60$ & $<40$ & \\
\hline$\leq 20$ & 4 & 1 & 5 \\
\hline $21-30$ & 27 & 24 & 53 \\
\hline $31-39$ & 46 & 61 & 107 \\
\hline Total (\%) & 78 (47.3) & 87 (52.7) & 165 (100) \\
\hline
\end{tabular}

in 87 patients $(52.12 \%)$. Although the prevalence was low, the pattern was similar to a study done by De La Sierra et al. in Spain which showed 30\% hypertriglyceridemia and $21.7 \%$ with low levels of high-density lipoprotein cholesterol. ${ }^{19}$ Similarly, in a study by $\mathrm{Xu} \mathrm{C}$ et al., the most common abnormal association was high TG and low HDL-C, followed closely by an increase in LDL-C.20 The result was high as compared to studies of $A$. BachirCherif et al. which showed increased Total Cholesterol combined with the increase in LDL levels the most common disorder (32.2\%). ${ }^{13}$

The prevalence of dyslipidemia has been found to be alarming. Its prevalence has been found to be increasing with age. So it may be useful to perform early mandatory screening, to facilitate early detection and timely intervention. In this context, the American Heart Association recommends that dyslipidemia screening should start when individuals are in their twenties and states that consistent monitoring is essential. ${ }^{21}$

Cardiovascular diseases are in rise in the developing countries like Nepal. It is linked to various controllable risk factors. Among these factors, dyslipidemia is often found to be one, which is related to the change in behavioral and dietary habits. ${ }^{22}$ The accelerated trend of diabetes and dyslipidemia deserves special attention. Early detection and effective management of diabetes and dyslipidemia is needed in young patients with high blood pressure and should be one of the public health priorities in our country.

We acknowledge that there are a few limitations in our study. Sample size is small, so it may not reflect the whole young population of country. Other traditional risk factors like BMI, dietary pattern, physical activity, family history has not been included in the study which might have contributed in high prevalence of diabetes and dyslipidemia. Different causes possible secondary hypertension were not analyzed. HBA1c and post prandial blood sugar also not included in the study.

\section{CONCLUSION}

Our study findings suggest that there is an alarming prevalence of diabetes, prediabetes and dyslipidemia among the young hypertensive patients which might serve as supportive data for health planners to formulate and implement policies that aim to increase public awareness about increasing prevalence of dyslipidemia and diabetes in young hypertensive patients. It also highlights the need of regular monitoring of blood glucose and lipid profile in young hypertensive individuals.

\section{CONFLICT OF INTEREST}

None declared.

\section{REFERENCES}

1. Onat A, Hergenç G, Sari I et al. Dyslipidemic hypertension: distinctive features and cardiovascular risk in a prospective population-based study. Am J Hypertens. 2005 Mar;18(3):409-16.

2. Wilson PW, D'Agostino RB, Parise $H$ et al...Metabolic syndrome as a precursor of cardiovascular disease and type 2 diabetes mellitus. Circulation. 2005 Nov 15; 112(20): 3066-3072.

3. Harrison T, Isselbacher K, Wilson J. Harrison's principles of internal medicine. 20th ed. New York: McGraw-Hill; 2019.

4. Gress TW, Nieto FJ, Shahar E et al. Hypertension and antihypertensive therapy as risk factors for type 2 diabetes mellitus: Atherosclerosis Risk in Communities Study. N Engl J Med. 2000; 342:905-912.

5. Boo S, Yoon YJ, Oh H. Evaluating the prevalence, awareness, and control of hypertension, diabetes, and dyslipidemia in Korea using the NHIS-NSC database: A cross-sectional analysis. Medicine (Baltimore). 2018. 97(51): e13713.

6. Kashyap Dahal, Prahlad Karki, Robin Maskey et al. Risk factors of acute myocardial infarction in young adults of Nepalese population. International Journal of Medicine in Developing Countries. 2018;2(3):80-85.

7. Chandra Mani Adhikari, Reeju Manandhar, Dipanker Prajapati et al. Acute ST-elevation myocardial infarction in young in Shahid Gangalal National Heart Centre, Kathmandu, Nepal. Journal of Advances in Internal Medicine. 2017; 06(02):27-31.

8. Chobanian AV, Bakris GL, Black HR et al. The seventh report of the joint national committee on prevention, detection, evaluation, and treatment of high blood pressure: the JNC 7 report. JAMA 2003; 289:2560-71.

9. National Institute of Health. 2012 Summer Accessed at Medlineplus. gov/magazine/lssues/summer12/articles/summer12pg6-7.html

10. American Diabetes Association Guidelines 2018. Classification and Diagnosis of Diabetes: Standards of Medical Care in Diabetes-2018. Diabetes Care. 2018 Jan;41(Suppl 1): S13-S27.

11. Meme N. Prevalence of undiagnosed diabetes and pre-diabetes amonghypertensive patients attending Kiambu district Hospital, Kenya: a crosssectionalstudy. Pan Afr Med J. 2015; 22:286.

12. Lüders $S$, Hammersen F. Diagnosis of impaired glucose tolerancein hypertensive patients in daily clinical practice. Int J ClinPract. 2005;59(6):632-8.

13. BachirCherif A, Bennouar S, Bouamra A et al. Prevalence of diabetes and dyslipidemia in hypertensive patients in the area of Blida (Algeria). Ann CardiolAngeiol (Paris). 2018 Jun;67(3):198203.

14. Huang XB. Prevalence of diabetes and unrecognized diabetes in hypertensive patients aged 40 to 79 years in southwest China. PLoS One 2017;12(2): e0170250. 
15. Thiombiano LP. Prevalence of dyslipidemia in the rural populationof Gueoul (Senegal). Ann CardiolAngeiol 2016;65(2):77-80.

16. Akintunde AA, Ayodele EO, Akinwusi OP. Dyslipidemia among newlydiagnosed hypertensives: pattern and clinical correlates. J Natl Med Assoc. 2010;102(5):403-7.

17. Zhang $X$. Prevalence of dyslipidemia and associated factors among thehypertensive rural Chinese population. Arch Med Res 2007; 38:432e439.

18. Pedrinellia R. LDL cholesterol and global risk stratification in referred hypertensive patients. Atherosclerosis 2005; 180:137-43.

19. De la Sierra A, Gorostidi M, Aranda P et al. Prevalence ofatherogenic dyslipidemia in Spanish hypertensive patients and its relationship with blood pressure control and silent organ damage. Rev Esp Cardiol2015; 68:592-8.

20. Xu C, Yang X, Zu S et al. Association between serum lipids, blood pressure, and simple anthropometric measures in an adult Chinese population. Arch Med Res. 2008;39(6):610-7.

21. Mosca L, Benjamin EJ, Berra K et. al. Effectiveness-based guidelines for the prevention of cardiovascular disease in women--2011 update: a guideline from the American Heart Association. Journal of the American College of Cardiology, 57(12), 1404-1423. American Heart Association.

22. Lopez AD. Global and regional burden of disease and risk factors, 2001: systematic analysis of population health data. Lancet. 2006:367(9524):1747-57. 